\title{
Protokoll der Jahresversammlung der Schweizerischen Entomologischen Gesellschaft vom 3. und 4. März 2017 am Agroscope Standort Changins
}

\author{
Matthias Borer ${ }^{1}$ \\ 1 Naturhistorisches Museum, Basel, Switzerland \\ http://zoobank.org/12B5EC74-C9FF-4441-9909-A09B1B7A1BAD \\ Corresponding author: Matthias Borer (matthias.borer@bs.ch)
}

Received 13 November 2017

Accepted 14 November 2017

Published 20 November 2017

Academic editor:

Thibault Lachat

Die Jahresversammlung der SEG wurde auf Einladung der eidgenössischen Forschungsanstalt für landwirtschaftlichen Pflanzenbau Agroscope Standort Changins am 3. und 4. März 2017 in Nyon durchgeführt. Der Freitagnachmittag war neben taxonomischen Beiträgen und der Präsentation eines interaktiven Bestimmungsschlüssels vor allem Themen der angewandten Entomologie in der Landwirtschaft gewidmet. Dr. Patrik Kehrli (Agroscope Changins) eröffnete die Tagung mit einem spannenden und facettenreichen Hauptvortrag über die Geschichte der Schädlingsbekämpfung. Patrik Kehrli führte von der Grünen Revolution über die Anfänge des IPM und deren Pioniere am Agroscope bis zu den diversen möglichen direkten und indirekten Methoden der Schädlingskontrolle. Zum Schluss gab er einen Ausblick auf die zukünftigen und neuen Methoden des IPM, wie den Einsatz von Drohnen, Nanotechnologie und vermehrt auch der Gentechnik.

Der Samstag war freien Themen aus der Faunistik, Systematik sowie der angewandten Entomologie gewidmet. Zudem wurde auch ein beeindruckendes Bildungsangebot für Schulklassen und Kindergruppen zum Thema Wildbienen präsentiert. Prof. Michel Chapuisat (Université Lausanne) eröffnete den zweiten Tag mit einem höchst spannenden Vortrag über Supergene und die Soziale Evolution bei Ameisen. Er stellte seine Forschung über die Gründe und mögliche Mechanismen für das Auftreten von monogynen und polygynen Waldameisenvölkern vor und verglich diese mit den Mechanismen bei der Roten Feuerameise.
Auf grosses Interesse stiess auch die am Ende der Tagung durchgeführte Führung durch die Insekten-Zucht in Changins, die in einem top modernen Minergie-Gebäude untergebracht ist. Serge Fischer und Stève Breitenmoser stellen einen Grossteil der während der Vortragsserie thematisierten Schädlinge und zum Teil auch deren Gegenspieler live vor. An der Generalversammlung vom Samstagmorgen nahmen 28 Mitglieder teil.

Agroscope Standort Changins, im Speziellen Stève Breitenmoser, sei für die hervorragende Organisation der Jahresversammlung und das Offerieren der Pausenverpflegungen herzlich gedankt.

\section{Generalversammlung}

\section{Begrüssung}

Der Präsident Dr. Stefan Ungricht eröffnet die Generalversammlung um 09:45 und begrüsst die 28 anwesenden Mitglieder.

\section{Protokoll der Generalversammlung 2016 in Neuchâtel}

Das Protokoll wird kommentarlos genehmigt. 


\section{Bericht des Präsidenten (Dr. S. Ungricht)}

\section{Administratives}

Für die Unterstützung im administrativen Bereich konnten wir 2016 wiederum auf das Centre Suisse de Cartographie de la Faune (CSCF) in Neuchâtel zählen. Die SEG ist Mitglied der Plattform Biologie der Akademie der Naturwissenschaften Schweiz (SCNAT) in Bern und wird da von der Geschäftsleiterin Pia Stieger betreut. www.naturwissenschaften.ch/organisations/bio

\section{Finanzielle Unterstützung}

Die Gesuche des Präsidenten um finanzielle Unterstützung wurden von den angefragten Institutionen vollumfänglich bewilligt. Es handelt sich dabei um folgende Beträge: Akademie der Naturwissenschaften Schweiz (SCNAT) in Bern: CHF 14'500.-, Biedermann-Mantel-Stiftung in Zürich: CHF 6'000.- und Syngenta AG in Basel: CHF 6'000.-. Wir sind allen drei Geldgebern für ihre wichtigen Beiträge dankbar.

\section{Jahresversammlung, Generalversammlung und Vor- standssitzungen}

Die traditionelle zweitägige Jahresversammlung, die unter der Bezeichnung entomo.ch allen Interessierten offen steht, wurde am Freitag, 4. und Samstag, 5. März 2016 am Muséum d'Histoire Naturelle Neuchâtel durchgeführt. Unsere lokalen Gastgeber waren Jessica Litman und Christophe Praz, denen für die Organisation des Anlasses ganz herzlich gedankt sei. Die Generalversammlung fand am Samstag, 5. März 2016 von 9:30 bis 10:30 am gleichen Ort statt. Die beiden regulären halbjährlichen Vorstandssitzungen fanden am Freitag, 4. März am Muséum d'Histoire Naturelle Neuchâtel und am Mittwoch, 2. November 2016 am Naturhistorischen Museum in Bern statt.

\section{Webseite}

Die Webseite unserer Gesellschaft sowie die OnlineAnmeldung zu unserer Jahrestagung entomo.ch wurde auch dieses Jahr wieder im Portal Naturwissenschaften Schweiz der Akademie der Naturwissenschaften Schweiz (SCNAT) geführt. Für die Betreuung danke ich Pascal Blanc (Chefredaktor) und Mariella Hobi (Support). www.naturwissenschaften.ch/organisations/seg

\section{Digitalisierung der Mitteilungen der Schweizerischen Entomologischen Gesellschaft}

Die in Zusammenarbeit mit der ETH-Bibliothek in Zürich digitalisierten Bände unserer Zeitschrift Mitteilungen der Schweizerischen Entomologischen Gesellschaft wurden im Verlaufe des Jahres auf der definitiven Plattform e-periodica publiziert. Damit sind unter Vorbehalt der beiden jeweils letzten Ausgaben (d.h. einer einjährigen Sperrfrist) alle Bände der Zeitschrift seit 1862 öffentlich zugänglich. Die digitalisierten Artikel bzw. deren Textinhalte werden auch durch Such- maschinen wie Google indiziert und bei entsprechenden Suchabfragen gelangt man damit direkt auf das Digitalisat. Ansprechpartnerin war für die SEG Regina Wanger, Leiterin des DigiCenters der ETH-Bibliothek in Zürich. www.e-periodica.ch

\section{Zukunft der Mitteilungen der Schweizerischen Ento- mologischen Gesellschaft}

Die einseitige Aufkündigung des Zeitschriften-Austauschprogramms mit ausländischen Institutionen seitens der ETH-Bibliothek sowie der Rücktritt des langjährigen Managing Editors Gerhard Bächli auf Ende 2016 war Anlass, die Zukunft unserer traditionsreichen Zeitschrift zu überdenken. Hannes Baur, der designierte Präsident für die Amtsperiode 2017-2020, hat dazu im Auftrag des Vorstandes eine Situationsanalyse vorgenommen und eine Offerte beim Verlag Pensoft eingeholt. Daraufhin wurde durch den aktuellen Präsident ein Statutenentwurf erarbeitet, der bei einer Annahme durch die Generalversammlung durchgreifende Änderung in der Erscheinungsweise, im Format, im Fokus und auch im Titel der Zeitschrift überhaupt erst ermöglichen würde.

\section{Dreiländertagung}

Die entomologische Dreiländertagung der DGaaE, ÖEG und der SEG findet am 13. bis 16. März 2017 in München bzw. Freising-Weihenstephan statt. Ein Schwerpunkt der Tagung sind Insekten auf Gehölzen bzw. die Forstentomologie. Weiterhin findet im Rahmen dieser Tagung das 25. Internationale Symposium zur Entomofaunistik in Mitteleuropa statt. www.dgaae.de/index.php/entomologentagung-2017.html

\section{Insekt des Jahres}

Ebenfalls in Zusammenarbeit mit Entomologen aus Deutschland und Österreich wurde für das Jahr 2017 die Gottesanbeterin (Mantis religiosa) zum Insekt des Jahres bestimmt. Zur Bewerbung dieser Insektenart wurde ein Poster und ein Flyer erarbeitet. www.naturwissenschaften. ch/service/news/84142-insekt-des-jahres-2017

Allen genannten Institutionen, Gremien und Personen sei hier nochmals ausdrücklich für ihre Unterstützung und ihren Einsatz im Namen des Vorstandes und der Mitglieder der Schweizerischen Entomologischen Gesellschaft gedankt!

\section{Bericht des Bibliothekars und der Redaktoren (Mitteilungen und Fauna Helvetica)}

\section{Bericht des Bibliothekars (Dr. P. Jeanneret)}

En 2016, à la suite du nouveau mode de fonctionnement de la bibliothèque de l'ETH qui renonce à stocker et envoyer des documents sous forme papier, le bulletin de la Société Suisse d'Entomologie n'a pas été envoyé aux 
partenaires d'échange. Ce changement correspond aussi à la volonté et la mise en place d'une nouvelle version, électronique, du bulletin. Selon les informations fournies par la bibliothèque de l'ETH, les publications reçues de la part des partenaires d'échange sont très peu nombreuses. Le bibliothécaire a été sollicité pour fournir des renseignements sur des publications dans le bulletin de la part de trois personnes en 2016.

\section{Bericht des Redaktors der Fauna Helvetica (PD Dr. D.} Burckhardt)

Im Berichtsjahr wurde kein Band publiziert, es sind aber mehrere Bände in Bearbeitung. Weit fortgeschritten sind die Bearbeitungen der Amphipoda, Vespidae, Cicadidae und Isopoda sowie eine Neubearbeitung der Bombus-Arten.

Mit den heutigen Möglichkeiten der elektronischen Publikation stellt sich die prinzipielle Frage, ob auch in Zukunft alle Bände von Fauna Helvetica in Papierform gerduckt werden sollen. Während dies bei Bestimmungsschlüsseln sicher weiterhin sinnvoll ist, empfehlen wir Checklisten und Verbreitungsatlanten fortan nur noch elektronisch zu publizieren. Dies ermöglicht Aktualisierungen und hilft, Produktions- und Lagerkosten zu reduzieren.

Wie immer klappte die Zusammenarbeit mit dem CSCF ausgezeichnet. Dafür möchte ich dem Leiter Dr. Y. Gonseth und seinen Mitarbeiterinnen und Mitarbeitern ganz herzlich danken.
Bericht der Redaktoren der Mitteilungen (Dr. G. Bächli, Dr. C. Germann und A. Sanchez)

Im Berichtsjahr 2016 erschien Band 89 unserer Mitteilungen in zwei Heften. Sie umfassten auf 294 Seiten 18 wissenschaftliche Artikel, 3 Buchbesprechungen (2 wurden nicht abgedruckt, sind jedoch im Inhaltsverzeichnis erwähnt!), das Protokoll der Jahresversammlung in Neuchâtel, den Nekrolog von Vittorio Delucchi, die Laudatio des Ehrenmitglieds Felix Amiet, die Gratulation zu Bernd Hausers 80igsten Geburtstag, die wissenschaftlichen Beiträge zur Tagung entomo.ch und die Jahresberichte unserer Sektionen und den Aufruf zum Prix Moulines.

Von den 18 wissenschaftlichen Artikeln waren 5 auf Deutsch, 3 auf Französisch und 10 auf Englisch.

Dr. Gerhard Bächli hat die Redaktionsleitung per Ende 2016 nach 24 Jahren sorgfältiger und kompetenter Tätigkeit als Redaktor der Mitteilungen abgegeben. In Zukun$\mathrm{ft}$ werden die MSEG in einem neuen Format erscheinen, welches sich noch in Abklärung befindet. Die verbleibenden Redaktoren danken Gerhard Bächli ganz herzlich für seine engagierte und zuverlässige Tätigkeit als Hauptredaktor.

\section{Berichte der Quästorin und der \\ Rechnungsrevisoren}

\section{Bericht der Quästorin (E. Leonetti)}

Frau Emanuela Leonetti legt den Anwesenden die Jahresrechnung 2016 vor. Daraus sind folgende Zahlen entnommen:

\begin{tabular}{|c|c|c|}
\hline Positionen / Objets & Ausgaben / Dépenses & Einnahmen / Revenus \\
\hline \multicolumn{3}{|l|}{ Publikationskosten / Charges de publications } \\
\hline Publications MSEG - impression, rédaction & $22^{\prime} 403.30$ & \\
\hline Publications MSEG - Digitalisation & 0.00 & \\
\hline Publication „Fauna Helvetica“ & 96.10 & \\
\hline Verwaltung / Administration & $8^{\prime} 416.80$ & \\
\hline Beitrag sc|nat / Cotisation sc/nat & $1^{\prime} 687.00$ & \\
\hline Arbeitsgruppenförderung / Groupes soutien de travail & $2^{\prime} 000.00$ & \\
\hline $\begin{array}{l}\text { Charges extraordinaires (bourses de soutien recherche } \\
\text { entomologique) }\end{array}$ & 542.83 & \\
\hline Verkauf Mitteilungen / Ventes bulletins & & $2^{\prime} 049.47$ \\
\hline Mitgliederbeiträge / Cotisations & & 16'194.02 \\
\hline Ventes Fauna Helvetica & & 9'175.82 \\
\hline Beiträge / Subventions: sc|nat & & $12 ' 000.00$ \\
\hline Syngenta & & $6^{\prime} 000.00$ \\
\hline Biedermann-Mantel-Stiftung & & $66^{\prime} 000.00$ \\
\hline Spenden und sonstige Einnahmen / Dons et autres produits & & 0.00 \\
\hline Zinsen / Intérêts: SEG-Konten / Comptes SEG & 38.20 & \\
\hline Fauna Helvetica-Konto / Compte Fauna Helvetica & & 0.00 \\
\hline Augmentation de réserve (résultat Fauna Helvetica) & 9.079 .72 & \\
\hline Totale / Totaux & $44 ' 263.95$ & $51 ' 419.31$ \\
\hline Jahresgewinn / Bénéfice annuel 2013 & & 7'155.36 \\
\hline
\end{tabular}


Die Erfolgsrechnung für das Jahr 2016 schloss mit einem Gewinn von CHF 7'155.36 (Vermögen CHF $\left.51^{\prime} 419.31\right)$.

\section{Bericht der Rechnungsrevisoren (C. Monnerat und Dr. M. Sartori)}

En tant qu'organe de contrôle de la société Entomologique Suisse, nous avons vérifié les comptes de l'exercice 2016.

Nous avons pu constater que:

- le bilan d'ouverture, le bilan de clôture et le compte d'exploitation correspondent à la comptabilité

- les justificatifs sont conformes, exacts et correspondent à l'activité habituelle de la SES

- la fortune de la société correspond aux compte à l'actif du bilan (liquidité + transitoires).

Pour sa part, la caissière assure que la tenue de la comptabilité a été faite, dans les limites de ses compétences, avec exactitude et selon les principes formulés dans le Code des Obligations (CO 959 t 662a/2).

C'est pourquoi nous prions l'assemblée d'accepter les comptes de l'exercice 2016 et de donner décharge à la caissière.

Der Quästorin wird einstimmig Decharge erteilt.

\section{Statutenrevision}

Hannes Baur fasst in einem Kurzvortrag mit dem Titel „Mitteilungen-Quo vadis?“ die wichtigsten Fakten zum geplanten Format der Mitteilungen zusammen. Detaillierte Unterlagen wurden vor der GV an alle Mitglieder versendet. Um das neue Format der Mitteilungen auch statutenkonform $\mathrm{zu}$ betreiben, sind einige kleine Anpassungen der Statuten notwendig.

Die vom Vorstand vorgeschlagene Statutenrevision wird einstimmig angenommen.

\section{Die Zukunft der Mitteilungen der Schweizerischen Entomologischen Gesellschaft}

Das vom Vorstand vorgeschlagene neue Format der Mitteilungen, neu Alpine Entomology, ist eine Open Access-Zeitschrift beim Pensoft-Verlag. Die akzeptierten Artikel werden sofort online publiziert und Ende Jahr kann eine Druckversion hergestellt werden. Die publizierten Daten werden elektronisch aufbereitet und stehen für jegliche weitere Arbeiten sofort und einfach zur Verfügung.

Das neue Format der Mitteilungen und der neue Name Alpine Entomology werden einstimmig angenommen.

\section{Budget und Mitgliederbeitrag}

\section{Budget 2017}

Der Präsident stellt das Budget für 2017 vor, das einen Gewinn von CHF 100.- vorsieht. Das Budget wird einstimmig genehmigt.

\section{Mitgliederbeitrag 2017}

Die Beiträge bleiben gleich: Mitglieder in der Schweiz CHF 60.-, Mitglieder im Ausland CHF 75.-; Studierende oder sich anderweitig in Ausbildung befindende Mitglieder zahlen während drei Jahren nur die Hälfte (CHF 30.-) des ordentlichen Beitrages.

\section{Vorstandswahlen für die Amtsperiode 2017-2020}

Hannes Baur, Kurator Entomologie am Naturhistorischen Museum der Burgergemeinde Bern und Spezialist für Erzwespen und Heuschrecken wird einstimmig zum neuen Präsidenten gewählt. Der bisherige Präsident Dr. Stefan Ungricht wird gemäss Statuten der neue Vizepräsident. Andreas Sanchez wird einstimmig als Managing Editor ad interim der Alpine Entomology gewählt. Alle übrigen Vorstandsmitglieder haben sich zur Wiederwahl zur Verfügung gestellt und wurden ebenfalls einstimmig in ihren Funktionen bestätigt.

\section{Personelles}

\section{Todesfall}

Dem 2016 verstorbenen SEG Mitglied Alfred Riggenbach (Phytopathologe, 1929-2016) wird mit einer Schweigeminute gedacht.

\section{Rücktritt}

Gerhard Bächli ist auf Ende 2016 aus dem Vorstand und als Managing Editor der Mitteilungen zurückgetreten. Während seiner 21-jährigen Funktion als Managing Editor hat er 43 Heften mit Total 7849 Seiten zur Publikation verholfen. Für seinen unermüdlichen und äusserst wertvollen Einsatz während mehr als zweier Jahrzehnte wird ihm grösster Dank ausgesprochen.

\section{Varia}

Veranstaltungshinweis: Vom 21.-23. September 2017 findet in Sevilla das „5. Encuentro Ibérico de Biología Subterránea" statt. Unter folgendem Link finden Sie alle wichtigen Informationen: https://5encuentrobssevill.wixsite.com/veibs 


\section{Prix Moulines 2017}

Die Jury hat sich entschieden, dass zwei besonders verdienstvolle Arbeiten für die Entomologie der Schweiz ausgezeichnet werden. Der Präsident gibt die Gewinner, Dr. Ladislaus Rezbanyai-Reser bzw. Hans-Peter Wymann und Christian Roesti bekannt. Der Prix Moulines ging in beiden Fällen an die Autoren von hervorragenden Fachbüchern, einerseits zu den Eulenfaltern der Schweiz und andererseits zu den Orthopteren der frankophonen Länder Europas.

HANS-PETER WYMANN, LADISLAUS REZBANYAI-RESER \& MAX HÄCHLER, unter Mitarbeit von Anne Luginbühl: Die Eulenfalter der Schweiz - Lepidoptera: Noctuidae, Pantheidae, Nolidae, 2015.
ÉRIC SARDET, CHRISTIAN ROESTI \& YOAN

BRAUD: Cahier d'Identification des Orthoptères de France, Belgique, Luxembourg \& Suisse, 2015.

\section{Jahresversammlung 2018}

Die nächste Jahresversammlung findet am 2. und 3. März 2018 am Naturhistorischen Museum der Burgergemeinde Bern statt.

Ende der Generalversammlung um 10:55

Basel, den 31. März 2017, der Sekretär Matthias Borer 